\title{
Ordenanza sobre animales de compañía en Cerdanyola del Vallés (Barcelona)
}

Elisabet Miras Lara ${ }^{1}$

El presente artículo pretende ser una muestra de cómo nuestro actual formato de vida ha obligado a regular el desarrollo de la vida de los animales en las ciudades, por lo que parece inevitable que las poblaciones españolas modernicen sus regulaciones municipales para garantizar la calidad de las relaciones. En consecuencia, surge la pregunta; "¿La convivencia de humanos y animales requiere de normativa que garantice la seguridad y bienestar de ambas partes?". Es sabido que existen reglas para poder vivir en sociedad, pero a veces estas reglas descuidan la inclusión de otros seres no humanos en nuestro medio. Por ello, quiero destinar este artículo a examinar la tarea legislativa que algunos entes municipales han desarrollado ante la convivencia con animales en sus ciudades, y concretamente hacerlo mediante el ejemplo que supone la ordenanza municipal de Cerdanyola del Vallés, que regula la tenencia de estos seres bastante correctamente ${ }^{2}$.

Esta orden se aprobó el 28 de Noviembre de 2002 por el pleno municipal, y regula la tenencia de animales domésticos y la propiedad de ciertos establecimientos que los contengan, así como la circulación y tenencia de perros potencialmente peligrosos. La ordenanza se estructura en diez títulos y abarca aspectos públicos y privados de la relación entre animales domésticos y sus propietarios o poseedores temporales.

\footnotetext{
${ }^{1}$ Licenciada en Derecho y estudiante del máster de Derecho Animal y Sociedad por La Universidad Autónoma de Barcelona. Voluntaria de la asociación protectora ADAC (Cerdanyola Del Vallès).

${ }^{2}$ Ordenanza municipal, 022003007423/2002, de 28 de Noviembre, "sobre tenencia de animales domésticos y de la conducción y tenencia perros considerados potencialmente peligrosos",
} (http://www.derechoanimal.info/bbdd/Documentos/1060.pdf). 
Estructuralmente, hablamos de una norma con 43 artículos, pero de contenido homogéneo. Encontramos tres grandes bloques; en la primera parte del texto se articulan las obligaciones que recaen sobre los propietarios y poseedores de animales domésticos o amansados y en el segundo, las implicaciones legales que supone poseer un animal catalogado como potencialmente peligroso. Finalmente, el tercer y último apartado describe las sanciones administrativas que corresponden al incumplimiento de la normativa.

Respecto al análisis del contenido, observamos como la legislación establece cuales son los animales susceptibles de quedar vinculados a la normativa i cuales no, con lo que el resultado es que, la orden incluye a todos los animales domésticos o asimilables, y por asimilables entiende todos los que se críen para el uso doméstico, por lo que hay una inclusión de todos los seres por ambigüedad. No obstante, el legislador se ocupa de excluir expresamente determinados animales, aunque no por sus características físicas o necesidades biológicas, sino por su finalidad no doméstica, es decir, excluye animales destinados a experimentación, alimentación o por estar en peligro de extinción ${ }^{3}$. Por tanto, mi conclusión al respecto es que, un mismo ratón puede ser destinado al ocio o a la experimentación, o que un ave exótica puede criarse para el deporte y estará sujeta a las garantías de ésta legislación, mientras que, el mismo pájaro destinado a la investigación no lo estará. Aun así, en favor de la ordenanza debo decir que, incluye una especial mención a los perros de vigilancia de obras de construcción, con lo que éstos también están sujetos a esta legislación pese a que no tengan un destino doméstico, esta regulación es tan garantista al respecto, que recoge incluso un programa previo de reinserción, preferente a su eutanasia, para cuando finalizan sus funciones de vigilancia.

\footnotetext{
${ }^{3}$ Ordenanza municipal, 022003007423/2002, de 28 de Noviembre, "sobre tenencia de animales domésticos y de la conducción y tenencia perros considerados potencialmente peligrosos", artículo 2, párrafo 10. (http://www.derechoanimal.info/bbdd/Documentos/1060.pdf).
} 
De la misma forma que se regulan las clases de animales vinculados por la normativa, la ordenanza municipal determina que sujetos pueden intervenir y que obligaciones tienen en relación a estos animales; por un lado, determina las concesiones y prohibiciones que tienen los propietarios, así como los poseedores, que también son responsables de la actuación de los animales en el periodo de posesión en el que estén bajo su responsabilidad. Paralelamente, se determinan las obligaciones de entes privados destinados a la comercialización de animales de carácter doméstico, así como las exigencias de los centros sin ánimo de lucro. Además de un tercer bloque destinado a los imperativos que vinculan al municipio como ente público, que como tal, debe prestar determinados servicios, entre ellos la recogida de los animales abandonados y el censo de todos los instalados en su demarcación. En cualquier caso, prohíbe a los tres grupos citados anteriormente el maltrato en la esfera privada, así como en exhibiciones públicas, la donación como premio en cualquier concurso, la venta de los animales a menores sin autorización previa de los tutores legales de estos, la venta para experimentación sin la correspondiente concesión administrativa o la venta ambulante de estos seres; supuestos que también penaliza la Ley Catalana de Protección de los Animales. Y finalmente, se prohíbe también el abandono de estos seres ${ }^{4}$. Sin duda, el artículo nueve de esta ordenanza supone una condena expresa que garantiza la seguridad jurídica.

En el título tercero, se engloban todas las posibles conductas de interés legal para el legislador municipal respecto de la esfera privada de los particulares que posean animales domésticos; se regula el alojamiento de los animales para garantizar unas condiciones de salubridad suficientes en los hogares, así como ciertas medidas de

\footnotetext{
${ }^{4}$ Ordenanza municipal, 022003007423/2002, de 28 de Noviembre, "sobre tenencia de animales domésticos y de la conducción y tenencia perros considerados potencialmente peligrosos", Artículo 9. (http://www.derechoanimal.info/bbdd/Documentos/1060.pdf).
} 
confort, ya que establece específicamente las medidas de las casetas de animales de más de 25 kilos, con lo que se garantiza la salud y el bienestar de éstos seres, sin dar lugar a la ambigüedad en la interpretación de la norma, precisamente gracias a que se especifican estas medidas de las instalaciones de alojamiento de los animales. También se incluye la prohibición de mantenerlos atados de forma permanente, de la misma forma que lo hace la Ley Catalana de Protección de los Animales ${ }^{5}$, así como la necesidad de estacionar los vehículos que contengan animales a la sombra de las vías y con ventilación permanente en el período de tiempo en el que la mascota se encuentre en el interior, y además, tampoco se permite la introducción de estos seres en maletas u objetos análogos por no contener ventilación suficiente. Respecto de los medios de sujeción de los animales en los elementos privativos de una vivienda, la administración también interviene para delimitar ciertas conductas, como es el caso del encadenado, que debe ser seguro para las personas pero también para los animales que se encuentren apresados. Igualmente, se imponen unos requisitos mínimos en la comunidad vecinal, ya que quienes tienen la potestad para restringir las relaciones en este medio son precisamente los mismos propietarios, por ello, estas normas mínimas solo hacen referencia al respeto de las horas nocturnas de descanso, sancionando la presencia de animales en patios y balcones durante la noche, aunque bajo la presunción de que estos animales se encontrarán en estas zonas el mínimo tiempo posible durante el día, para evitar molestias graves al resto de la comunidad de propietarios.

Singularmente, este apartado contiene una detallada estructura de cómo estos seres deben interactuar en la vía pública. Es fundamental que vayan atados con correa, tengan un collar identificativo alrededor del cuello, que su dueño lleve la identificación

\footnotetext{
${ }^{5}$ Decreto Legislativo, 2/2008, de 15 de Abril, "por el que se aprueba el Texto refundido de la Ley de protección de los animales", artículo 5.L. Prohibiciones, (http://www.derechoanimal.info/bbdd/Documentos/458.pdf).
} 
censal ${ }^{6}$ in situ durante las salidas con el animal por la vía pública, así como disponer de bozal, hablemos o no de un animal potencialmente peligroso, bozal que deberá colocársele al animal cuando la autoridad correspondiente lo considere necesario. Además, se determina como identificar un animal vagabundo; que solo será aquel que no tenga propietario aparente ni identificación posible, en este caso, el animal podrá ser eutanasiado sino se consigue la reinserción previa ${ }^{7}$. Debo decir, que el posible sacrificio citado en varias ocasiones en la ordenanza, debe limitarse a los casos que la Ley Catalana de Protección de los Animales permite, ya que a diferencia del resto de España la normativa autonómica de esta comunidad es altamente proteccioncita. De todos modos, es cierto que la Ley Catalana regula de forma superficial la delicada materia de la que hablamos. Primeramente, no define el sacrificio como eutanasia ${ }^{8}$, que es precisamente una nomenclatura que utiliza el legislador en otras normativas que también regulan la posesión y el uso de animales, como es el caso de la regulación sobre experimentación con estos seres, y con estos términos dignificar el trato del animal y anormalizar esta práctica, y segundo, porque no hay una condena expresa del método por parte del legislador que demuestre cuan residual es esta técnica. Además, la regulación de la casuística sobre la eutanasia legal se remite a la vía reglamentaria. Con lo que, actualmente lo que tenemos es un breve Decreto sobre los métodos de eutanasia legalizados y su uso en animales abandonados ${ }^{9}$. Y por tanto, el uso inapropiado de los términos que definen el sacrificio y la remisión normativa de la casuística a otro texto legal; producen un resultado de inseguridad jurídica sobre la

\footnotetext{
${ }^{6} \mathrm{AIAC}$, archivo de identificación de animales de compañía, Consejo de Colegios de Veterinarios de Catalunya.

${ }^{7}$ Ordenanza municipal, 022003007423/2002, de 28 de Noviembre, "sobre tenencia de animales domésticos y de la conducción y tenencia perros considerados potencialmente peligrosos", artículo 16.

8 Real Decreto 53/2013, de 1 de febrero, "por el que se establecen las normas básicas aplicables para la protección de los animales utilizados en experimentación y otros fines científicos, incluyendo la docencia", artículo 3.1.c "definiciones, eutanasia". (http://www.derechoanimal.info/bbdd/Documentos/1029.pdf).

${ }^{9}$ Real Decreto 254/2000, de 24 de julio, "por el que se establecen los métodos de eutanasia para los animales de compañía que se tienen que sacrificar", (http://www.derechoanimal.info/bbdd/Documentos/454.pdf).
} 
interpretación del sacrificio válido, que según los intereses de cada sujeto se hará de forma más o menos flexible. Este problema tiene fácil solución, la refundición de la legislación que implica a los animales domésticos, ya que tienen un especial trato en nuestra sociedad. Todo este razonamiento viene dado porque, a mi juicio, la normativa sobre el sacrificio de los animales de compañía es, en realidad, la regulación de la vida en su vertiente negativa, que al fin y al cabo, es un principio supremo entre nuestros valores sociales y legales.

Por otro lado, se regula como deben trasladarse los animales domésticos dentro de los transportes públicos o en las instalaciones periféricas de estos. Deben estar confinados o no según dispongan las regulaciones vigentes de cada tipo de transporte, lo cual es también muy ambiguo y crea, de nuevo, inseguridad para el ciudadano de a pie. Excepcionalmente, si son perros guía, la ordenanza cita que podrán circular libremente en los medios de transporte público para conducir a sus propietarios en estos emplazamientos. Pero, en cualquier caso, debemos atenernos a la normativa que tenga cada medio de transporte, por tanto; autobús, metro, tren, etc., tendrán reglas distintas pese a ser de la misma comunidad autónoma.

Respecto de las instalaciones privadas, encontramos dos bloques distintos de legislación, en la primera sección, se prohíbe directamente la entrada de animales en ellos, serán: comercios alimentarios, parques de juegos infantiles y piscinas. $Y$ en el segundo, se engloba el resto de locales, en los que se dará la potestad a los propietarios de elegir si consienten o no la entrada de animales domésticos en sus establecimientos. Una buena estrategia de inclusión del sector de la población que pose animales de compañía en la vida pública sería, recomendar o incluso incentivar económicamente, en las propias ordenanzas municipales la entrada de perros y gatos 
en determinados locales y restaurantes, como si se está haciendo en países pioneros como Alemania o Suiza ${ }^{10}$.

Sobre las vías de peatones, la circulación es libre y la administración solo interviene respecto a la recogida de las deposiciones fecales, que deberán arrojarse solamente en los contenedores domiciliarios o en los que la autoridad municipal lo permita. Tampoco se podrá alimentar ni asear a los animales en estas vías ${ }^{11}$. Y respecto a la circulación de vehículos a motor, con animales dentro, por las carreteras públicas, la ordenanza se remite a las normas de circulación nacional y recomendaciones de la dirección general de tráfico. Llegados a este punto, el problema con el que nos encontramos es el siguiente, la materia no está desarrollada en la ordenanza, es decir, no se puntualizan cuales son los medios homologados para el atado de los animales durante el transporte en un vehículo privado, pero es que observando las normas de circulación a las que nos remite, el ciudadano se encuentra igualmente con problemas de aplicación; primeramente, existe un gran abanico de legislaciones con citas a la conducción con animales en vehículos y segundo, que la regulación es ambigua. Con lo que sería mucho más fácil que la administración municipal examinase la normativa nacional y autonómica y dispusiera lo que es legal en su término municipal. Para ejemplificar la pluralidad normativa voy a citar solamente dos normas de rango distinto para mostrar la dispersidad con la que se encuentra el ciudadano; por un lado, existe la Ley Catalana de Protección de los Animales, que en su artículo 8 regula el

\footnotetext{
${ }^{10}$ Borja Domínguez Fernández, 30 de agosto de 2012, 08:00 horas, desde http://smoda.elpais.com/articulos/vivesen-una-ciudad-dog-friendly/2245

11 Ordenanza municipal, 022003007423/2002, de 28 de Noviembre, "sobre tenencia de animales domésticos y de la conducción y tenencia perros considerados potencialmente peligrosos", artículo 22. (http://www.derechoanimal.info/bbdd/Documentos/1060.pdf).
} 
"traslado de animales"12, por su contenido, parece que sea un precepto destinado a los usuarios del transporte de animales de ganadería, aunque no debemos presuponer la exclusión de los domésticos porque el legislador no lo especifica propiamente. Por otro lado, el ciudadano se encuentra con la legislación nacional sobre circulación, que en su artículo 18 del reglamento de circulación de vehículos a motor, nos dice, y cito textualmente; "El conductor de un vehículo está obligado a mantener su propia libertad de movimientos, el campo necesario de visión y la atención permanente a la conducción, que garanticen su propia seguridad, la del resto de los ocupantes del vehículo y la de los demás usuarios de la vía. A estos efectos, deberá cuidar especialmente de mantener la posición adecuada y que la mantengan el resto de los pasajeros, y la adecuada colocación de los objetos o animales transportados para que no haya interferencia entre el conductor y cualquiera de ellos. ${ }^{\prime 13}$ Visto el articulado es fácil concluir la ineficacia que se desprende de una regulación dispersa en el ordenamiento jurídico y la ambigüedad de este último texto, por tanto, el legislador municipal debería ser más preciso y no remitirse a los términos de éste Reglamento Estatal. En mi opinión, sería conveniente que los entes locales recomendasen ciertos medios de seguridad expresamente en sus ordenanzas, y orientar así, a los propietarios de cómo deben transportar al animal ya des de la legislación más cercana; pero sin que ello sea obligatorio para los ciudadanos, porque entiendo que si así fuese, cada ciudad pondría unos medios imperativos distintos y ello provocaría el rechazo de la norma en una sociedad en constante movimiento globalizado, por lo que entiendo, que solo debe ser una nota informativa, una guía para el propietario del animal, evitando así la consecuencia contraria a lo deseado, porque la finalidad última es

\footnotetext{
12 Decreto Legislativo, 2/2008, de 15 de Abril, "por el que se aprueba el Texto refundido de la Ley de protección de los animales", artículo 8.1. Traslado de animales. (http://www.derechoanimal.info/bbdd/Documentos/458.pdf).

13 Real Decreto 1428/2003, de 21 de noviembre, "por el que se aprueba el Reglamento General de Circulación para la aplicación y desarrollo del texto articulado de la Ley sobre tráfico, circulación de vehículos a motor y seguridad vial", artículo 17, (Boletín Oficial del Estado).
} 
proteger al conductor pero también al animal del riesgo que supone la circulación en vehículos a motor, y por tanto, la obligatoriedad de la norma solo provocaría la dificultad de aplicación con las diferencias posibles en cada municipio y finalmente, la no aplicación por falta de uniformidad de la legislación, pese al riesgo de ser sancionado por la autoridad municipal.

El siguiente título, abarca las directrices a tener en cuenta sobre los animales que se encuentren en núcleos zoológicos, ya sean criaderos, centros veterinarios o de investigación. Básicamente, es una normativa que refuerza la necesidad de salubridad de todos estos núcleos durante la manipulación de estos animales en propiedad. Pero si algo hay que destacar muy positivamente es, la condena simbólica, mediante la prohibición expresa de usar animales de laboratorio para experimentación sin anestesia y su correspondiente cura obligatoria posterior por el daño causado en su integridad física, es pues, una condena aplaudible pero emblemática, porque la legislación estatal si permite la experimentación sin anestesia cuando la investigación lo requiera ${ }^{14}$.

Finalmente, el capitulo octavo y noveno recogen, respectivamente, especificidades sobre perros y gatos y documentación municipal más restrictiva. Entre otros temas, la ordenanza dispone que, para perros potencialmente peligrosos, se realizará un especial seguimiento por la administración municipal. Estos perros deben cumplir unas características físicas o de raza, para clasificarse como agresivos y en consecuencia, quedarán vinculados a esta normativa más restrictiva, pero, de nuevo la legislación estatal, autonómica y municipal regulan la materia de forma distinta. La realidad es que la normativa nacional establece solamente ocho razas de obligada clasificación

\footnotetext{
${ }^{14}$ Real Decreto 53/2013, de 1 de febrero, "por el que se establecen las normas básicas aplicables para la protección de los animales utilizados en experimentación y otros fines científicos, incluyendo la docencia", artículo 26.1.b “anestesia y analgesia". (http://www.derechoanimal.info/bbdd/Documentos/1029.pdf).
} 
como peligrosas $^{15}$, que las comunidades deben incluir en sus legislaciones, pero además, cada territorio tiene potestad para ampliar respecto de otras razas que también consideren que lo son ${ }^{16}$. Asimismo, la propia Ordenanza de Cerdanyola del Vallés incluye tres razas más de animales de las que la Ley Catalana sobre Tenencia de Perros Potencialmente Peligrosos prevé ${ }^{17}$. Sin olvidar, la previsión existente en las tres normas citadas de poder incluir todo aquel animal que por rasgos físicos o de comportamiento muestre este carácter de agresividad. Con lo que, a la práctica, tenemos unas diferencias normativas importantes que afectan a las fronteras autonómicas pero también municipales.

En cualquier caso, en Cerdanyola del Vallés, se necesita de una licencia especial renovable, de cinco años de durada para perros potencialmente peligrosos. Este último precepto permite garantizar la seguridad jurídica de las personas, pero dudo también si la de los animales, ya que es un artículo que ejemplifica el rango legal de "cosa" que tiene el animal para la administración, porque se consolida en la regulación como una tasa, como si de cualquier bien material se tratase, y pese al reconocimiento expreso que hace la Ley Catalana de Protección de los Animales domésticos, en un intento de desvincularlos de esta categoría, las instituciones públicas mantienen la ordenación como bien mueble respecto de todos los animales domésticos

\footnotetext{
${ }^{15}$ Real Decreto, 287/2002, de 22 de marzo, "por el que se desarrolla la Ley 50/1999, sobre el régimen jurídico de la tenencia de animales potencialmente Anexo I. (http://www.derechoanimal.info/bbdd/Documentos/211.pdf).

${ }^{16}$ Ley 10/1999, de 30 de julio, "sobre la tenencia de perros considerados potencialmente peligrosos" artículo 1.c. "definiciones", (http://www.derechoanimal.info/bbdd/Documentos/462.pdf).

${ }^{17}$ Ordenanza municipal, 022003007423/2002, de 28 de Noviembre, "sobre tenencia de animales domésticos y de la conducción y tenencia perros considerados potencialmente peligrosos", artículo 32.1.c. (http://www.derechoanimal.info/bbdd/Documentos/1060.pdf).
} 
potencialmente peligrosos al concebirlos solamente como una tasa en su ordenación ${ }^{18}$. Para ser más concretos, El Decreto Legislativo que recoge el texto refundido de la Ley de Protección de los animales, ya en el preámbulo indica una posición descosificada hacia estos seres, y este reconocimiento se culmina en la redacción del capítulo II de la ley, sobre "normas generales de protección de los animales"19. En este caso, el legislador pone límites a la propiedad introduciendo prohibiciones, obligaciones y controles a los dueños de estos animales. En la misma línea, encontramos la redacción del artículo 511-1, párrafo tercero, del Código Civil Catalán; en el que se reconoce expresamente la no consideración de cosas a los animales ${ }^{20}$. Por tanto, mi conclusión respecto de estas dos normas es muy positiva, ya que hay un reconocimiento singular de los animales respecto del resto de bienes, entiendo por eso, un reconocimiento negativo de la Ley Catalana al excluirlo de la categoría propiamente de cosas, pero no definir una nueva categoría para estos seres. En cualquier caso, el tema que nos atañe es precisamente la existencia de un reconocimiento negativo, que sin duda vincula al derecho civil y administrativo, por lo que considero, que la administración catalana a todos sus niveles, debería actualizar estos términos, es decir, bajo el principio de administración única ${ }^{21}$, entiendo que la línea legislativa debe ser la misma a todos los niveles, y por tanto, la obligación al respecto, incluye nuestra Ordenanza municipal, que precisamente, no está encaminada hacia un reconocimiento autónomo de los animales como una categoría separada del resto de cosas legalmente apropiables, especialmente aquellos animales domésticos clasificados como potencialmente

\footnotetext{
${ }^{18}$ Ordenanza municipal, 022003007423/2002, de 28 de Noviembre, "sobre tenencia de animales domésticos y de la conducción y tenencia perros considerados potencialmente peligrosos", artículo 33.2. (http://www.derechoanimal.info/bbdd/Documentos/1060.pdf).

${ }^{19}$ Decreto Legislativo, 2/2008, de 15 de Abril, "por el que se aprueba el Texto refundido de la Ley de protección de los animales", capítulo II "sobre normas generales de protección de los animales". (http://www.derechoanimal.info/bbdd/Documentos/458.pdf).

${ }^{20}$ Código Civil de Catalunya, artículo 551.c, apartado 3‥ (DOGC número 4640 de 24/5/2006).

${ }^{21}$ Constitución Española de 1978, Título VIII, Capítulo III, Artículo 149.1
} 
peligrosos, ya que los contempla como una tasa administrativa, es decir, un bien por el que pagas una precio para mostrar tu propiedad al respecto frente a la autoridad vigente; por tanto, la administración local no contribuye a desvincular los animales de la categoría de cosas, ya que se les sigue poniendo un valor económico, calificación que los sujetos, a diferencia de los objetos, en principio no tienen.

También el propietario deberá cumplir unos requisitos previos para poseer estos animales, entre ellos: ser mayor de edad, sin antecedentes penales, acreditar la capacidad psicológica mediante un informe médico o no haber sido sancionado por infracciones de la ley nacional y autonómica que estipula las especificidades de la tenencia de animales potencialmente peligrosos ${ }^{22}$. Respecto de su participación en las vías públicas del municipio deberán llevar siempre una correa de menos de 2 metros de extensión y bozal homologado.

En el capitulo noveno, se editan algunas licencias administrativas extraordinarias respecto de las habituales para los centros. Cabe destacar, la necesidad de incluir a los particulares que crían camadas en sus hogares y los poseedores de animales de distintas especies, que deben inscribirse como núcleos zoológicos; así como entregar la correspondiente notificación previa al ayuntamiento de la instalación de carpas de circos en su demarcación, para hacer los correspondientes controles de seguridad y protección de los animales que estos tengan ${ }^{23}$; disposición ciertamente adecuada, teniendo en cuenta la falta de legislación específica en materia de circos y carpas ambulantes con espectáculos de animales.

\footnotetext{
${ }^{22}$ Ordenanza municipal, 022003007423/2002, de 28 de Noviembre, "sobre tenencia de animales domésticos y de la conducción y tenencia perros considerados potencialmente peligrosos", artículo 34. (http://www.derechoanimal.info/bbdd/Documentos/1060.pdf).

${ }^{23}$ Ordenanza municipal, 022003007423/2002, de 28 de Noviembre, "sobre tenencia de animales domésticos y de la conducción y tenencia perros considerados potencialmente peligrosos”, artículo 37.1, párrafo 20. (http://www.derechoanimal.info/bbdd/Documentos/1060.pdf).
} 


\section{dA derecho ANIMAL la web center de los animales con derecho}

Por tanto, después de analizar minuciosamente la legislación municipal de Cerdanyola del Vallés, puedo afirmar racionalmente que; si es necesaria una regulación municipal actualizada sobre animales domésticos y centros de cría, experimentación o exposición de animales en cada municipio, porque la interrelación de personas y animales se complica en el desarrollo diario de la vida en las ciudades; y requerimos respecto de estas rutinas, unos mínimos de convivencia regulados que garanticen la seguridad y protección de ambas partes. Pues contemplo que, de la misma forma que ascendimos a mujeres y niños al rango legal y capacidad del hombre civilizado occidental a finales del siglo XIX ${ }^{24}$, otorgando por ejemplo, el derecho al voto a las mujeres o la creación de los derechos de la infancia para los niños, porque no reafirmar un estatus intermedio para los animales domésticos, desde la legislación local, completando el reconocimiento existente para estos seres en la legislación autonómica de rango superior. En definitiva, las ordenanzas, son las reglas más cercanas al ciudadano, son las normas con las que más exigentes podemos ser, por ello, creo que debemos aprovechar esta proximidad, para mejorar la protección legal de los animales domésticos en el desarrollo de sus vidas en nuestras ciudades.

\footnotetext{
${ }^{24}$ Unicef 2013, 11:58 horas, desde http://www.unicef.org/spanish/rightsite/sowc/pdfs/panels/SOWC\%20all\%20panels\%20SP.pdf
} 